\title{
APLICAÇÃO DA ESCALA EM AVALIAÇÃO DE RISCO PARA O DESENVOLVIMENTO DE LESÕES DECORRENTES DO POSICIONAMENTO CIRÚRGICO DO PACIENTE
}

\author{
Application of the risk assessment scale for the development \\ of injuries due to surgical positioning \\ Aplicación de la escala en la evaluación de riesgos para el desarrollo de \\ lesiones derivadas del posicionamiento quirúrgico del paciente

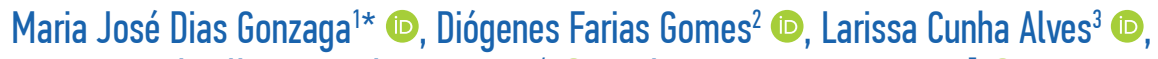 \\ Mikaelle Fernandes Marques ${ }^{4} \mathbb{C}$, Raila Souto Pinto Menezes ${ }^{5}$ (])
}

RESUMO: Objetivo: Identificar se há riscos consecutivos do posicionamento cirúrgico, por meio da aplicação da Escala de Avaliação de Risco para o Desenvolvimento de Lesões Decorrentes do Posicionamento Cirúrgico (ELPO). Método: Estudo observacional, descritivo, transversal, com abordagem quantitativa, executado em um hospital de ensino do norte do estado do Ceará. Foram utilizados dois intrumentos, um de perfil clínico-epidemiológico com base em entrevista estruturada e a ELPO, aplicada no período perioperatório a 31 pacientes cirúrgicos. Resultados: A maioria dos pacientes era do sexo biológico masculino, parda, na faixa etária entre 18 e 39 anos. Os procedimentos mais frequentes foram debridamentos e reduções de fraturas, em conformidade com as internações por acidentes motociclísticos. Quando avaliados quanto ao risco de lesões decorrentes do posicionamento cirúrgico, todos os pacientes apresentaram escore ELPO $\leq 19$, isto é, em menor risco para o desenvolvimento de lesões. Conclusão: O estudo oportunizou conhecer o perfil dos pacientes submetidos a procedimentos cirúrgicos na instituição, assim como a caracterização dos participantes quanto ao risco de desenvolver lesões decorrentes da posição cirúrgica, que foi classificado como baixo.

Palavras-chave: Posicionamento do paciente. Lesão por pressão. Cuidados de enfermagem.

ABSTRACT: Objective: To identify whether there are consecutive risks of surgical positioning through the application of the Risk Assessment Scale for the Development of Injuries due to Surgical Positioning (ELPO). Method: Observational, descriptive, cross-sectional study with a quantitative approach, carried out in a teaching hospital located in the north of the state of Ceará, Brazil. Two instruments were used, a clinical-epidemiological profile based on a structured interview and the ELPO scale, applied in the perioperative period to 31 surgical patients. Results: Most patients were men, mixed-race, and aged between 18 and 39 years. The most frequent procedures were debridement and fracture reduction, in accordance with hospitalizations due to motorcycle accidents. When evaluating the patients as for the risk of injuries due to surgical positioning, all subjects had ELPO score $\leq 19$, that is, they presented a lower risk for the development of injuries. Conclusion: The study allows for the profile of patients undergoing surgical procedures in the institution to be known, as well as the characterization of the participants regarding the risk of developing injuries due to surgical positioning, which was classified as low.

Keywords: Patient positioning. Pressure ulcer. Nursing care.

\footnotetext{
'Mestranda em Enfermagem na Saúde do Adulto na Universidade de São Paulo - São Paulo (SP), Brasil. Especialista em Urgência e Emergência com caráter de Residência pelo Centro Universitário INTA (UNINTA) - Sobral (CE), Brasil.

2Doutorando em Saúde Coletiva pela Universidade Estadual do Ceará (UECE) - Fortaleza (CE), Brasil.

${ }^{3}$ Especialista em Enfermagem Médico-Cirúrgica com área de concentração em Centro Cirúrgico, Recuperação Pós-Anestésica e Central de Material e Esterilização. Docente do UNINTA - Sobral (CE), Brasil. ¿Mestranda em Saúde da Família pelo Programa de Pós-Graduação em Saúde da Família da Universidade Federal do Ceará (UFC) - Fortaleza (CE), Brasil.

${ }^{5}$ Mestre em Saúde da Família pela Universidade Estadual Vale do Acaraú (UVA). Tutora do Programa de Residência em Urgência e Emergência do UNINTA. Docente do curso de Enfermagem no UNINTA Sobral (CE), Brasil.

${ }^{*}$ Autora correspondente: maiserdias@hotmail.com

Recebido: 31/05/2020 - Aprovado: 31/03/2021

https://doi.org/10.5327/Z1414-4425202100020006
} 
RESUMEN: Objetivo: Identificar el riesgo, mediante la aplicación de la Escala de Evaluación de Riesgos para el Desarrollo de Lesiones Derivadas del Posicionamiento Quirúrgico (ELPO). Método: Estudio observacional, descriptivo, transversal con abordaje cuantitativo, realizado en un hospital universitario del norte del estado de Ceará. Se utilizaron dos instrumentos, uno con perfil clínico-epidemiológico basado en entrevista estructurada y el ELPO, aplicado en el período perioperatorio a 31 pacientes quirúrgicos. Resultados: La mayoría de los pacientes eran hombres biológicos, pardos, con edades entre 18 y 39 años. La mayor frecuencia de procedimientos fue el desbridamiento y reducción de fracturas, en consenso con las hospitalizaciones por accidentes de motocicleta. Cuando se evaluó el riesgo de lesiones por posicionamiento quirúrgico, todos los pacientes tuvieron un puntaje ELPO $\leq 19$, es decir, un menor riesgo de desarrollar lesiones. Conclusión: El estudio permitió conocer el perfil de los pacientes sometidos a procedimientos quirúrgicos en la institución, así como la caracterización de los participantes en cuanto al riesgo de desarrollar lesiones derivadas de la posición quirúrgica, el cual fue clasificado como bajo.

Palabras clave: Posicionamiento del paciente. Úlcera por presión. Atención de enfermería.

\section{INTRODUÇÃ̃O}

A segurança do paciente constitui um dos grandes desafios dos cuidados de saúde nos dias atuais, que vêm sendo amplamente discutidos nos últimos tempos, gerando debates em âmbito mundial. Entretanto, ainda há muitas metas a serem alcançadas com o intuito de melhorar a qualidade da assistência ${ }^{1}$.

Nesse sentido, a qualidade do cuidado está diretamente atrelada à segurança do paciente. Esta envolve a prevenção de erros no cuidado prestado ao paciente e a eliminação de danos que podem ser causados por tais erros. Os acidentes adversos no âmbito hospitalar ocasionados ao paciente podem resultar em danos graves, gerando morbidade ou mortalidade ${ }^{2}$. Com base nas metas internacionais para segurança do paciente, criou-se o protocolo de cirurgia segura.

O Centro Cirúrgico (CC) é considerado uma área crítica no ambiente hospitalar, em que são desenvolvidos procedimentos cirúrgicos de várias complexidades e que constitui um dos setores propícios a oferecer risco e gerar inúmeros casos de eventos adversos (EA) de hospitalização ${ }^{3}$.

Durante a internação no CC, o paciente perpassa os seguintes períodos que compõem a fase da experiência cirúrgica: pré-operatório imediato, transoperatório (que inclui o intraoperatório) e pós-operatório imediato (que inclui a recuperação anestésica).

No período perioperatório, são diversos os fatores que podem impulsionar um EA, como procedimentos que superam duas horas e que podem comprometer a oxigenação dos tecidos; hipotermia, que pode causar prejuízos na perfusão de todo o corpo, prejudicando a oxigenação e favorecendo o surgimento de lesões; posicionamento do paciente e ausência de alívio de pressão5.

O enfermeiro, em sua prática profissional no CC, pode identificar o paciente com risco de lesão por posicionamento cirúrgico no período intraoperatório. Trata-se do "risco de mudanças anatômicas e físicas involuntárias, resultantes de postura ou equipamento usado durante procedimento invasivo/ cirúrgico" e que tem os seguintes fatores de risco: desorientação, edema, emaciação, imobilização, fraqueza muscular, obesidade e distúrbios sensoriais perceptivos decorrentes da anestesia ${ }^{6}$.

Ao posicionar o paciente na mesa operatória, existe uma ferramenta para detectar o risco de uma complicação pós-operatória, a lesão por pressão, que é a Escala de Avaliação de Risco para o Desenvolvimento de Lesões Decorrentes do Posicionamento Cirúrgico do Paciente (ELPO). Esse instrumento foi validado e desenvolvido no Brasil no ano de 2013 pela doutora em Enfermagem Camila Mendonça de Moraes Lopes, com o propósito de nortear a prática clínica do enfermeiro perioperatório. Foi desenvolvido na tese de doutorado "Escala de avaliação de risco para o desenvolvimento de lesões decorrentes do posicionamento cirúrgico: construção e validação"”.

O propósito da ELPO é guiar a prática clínica, auxiliando o enfermeiro na tomada de decisões sobre o cuidado prestado ao paciente cirúrgico, principalmente na prevenção de possíveis complicações relacionadas ao posicionamento cirúrgico. Isso se torna fundamental no processo de trabalho de enfermagem, sendo um instrumento prático em sua aplicação e que deve ser executado ao se posicionar o paciente na mesa operatória ${ }^{7,8}$.

A ELPO considera os fatores de riscos baseados nas melhores evidências disponíveis, a saber: tipo de posição cirúrgica, tipo de anestesia, tempo cirúrgico, tipo de superfície de suporte, posição dos membros e fatores relacionados ao paciente, como idade e comorbidades, com o objetivo de obter uma acomodação adequada, ausência de riscos e execução de um procedimento cirúrgico seguro e eficiente ${ }^{7}$.

O advento da ELPO só vem a somar informações e indicar atenção especial àqueles pacientes que possam estar em situação de maior risco e necessitem de cuidados especiais, pois o escore varia de sete a 35 pontos. Quanto maior o escore, maior o risco de o paciente desenvolver lesões relacionadas ao posicionamento cirúrgico ${ }^{7,8}$. 
As posições básicas que existem são decúbito dorsal ou posição supina, decúbito ventral ou posição prona e posição lateral. Elas podem ser modificáveis de acordo com o tipo de cirurgia, a equipe anestésico-cirúrgica ou as especificidades do paciente. Entre as modificações mais conhecidas estão as posições: Trendelenburg, Trendelenburg reversa, litotômica, posição sentada ou Fowler modificada, Kraske ou Jacknife e outras ${ }^{9,10}$.

O enfermeiro, como profissional responsável pelo paciente no CC, provê cuidados diretos, garantindo sua proteção e segurança ao valer-se da Sistematização da Assistência de Enfermagem Perioperatória (SAEP), ferramenta disponível, indispensável e de alta importância que permite melhor avaliação, bem como assistência totalitária de acordo com as necessidades de cada paciente ${ }^{5}$.

Em vista das declarações, surgiu o seguinte questionamento: os pacientes submetidos ao procedimento cirúrgico correm o risco de desenvolver lesão decorrente do posicionamento?

A pergunta norteadora surgiu da vivência como profissional residente no CC em um hospital de ensino, visto que seria necessária a aplicação do instrumento de avaliação no paciente no período intraoperatório, identificando se há riscos consecutivos do posicionamento cirúrgico. Logo, este estudo é voltado aos pacientes cirúrgicos em relação ao risco de desenvolvimento de lesão por pressão, para os quais o enfermeiro do centro cirúrgico pode estratificar o cuidado com maior necessidade de prevenção em todos os processos assistenciais.

Este estudo fez-se necessário por ser o hospital da pesquisa referência no atendimento de urgências e emergências ("porta aberta") e portador de índices elevados de procedimentos cirúrgicos, havendo necessidade de aplicação da ELPO, que possibilita identificar os riscos de surgimento de lesões por pressão. Além disso, é válido mencionar que a pesquisa pretende contribuir para nortear as ações de cuidados, promover segurança ao paciente e o desenvolvimento de educação permanente no serviço a fim de melhorar a qualidade da assistência de enfermagem e incentivar o desenvolvimento de protocolos de cuidados direcionados para o posicionamento cirúrgico do paciente.

\section{OBJETIVO}

Identificar o risco de lesão por pressão em pacientes cirúrgicos por meio da aplicação da ELPO.

\section{METódos}

Trata-se de um estudo observacional, descritivo, transversal, com abordagem quantitativa. A pesquisa foi desenvolvida de acordo com os preceitos éticos da Resolução do Conselho Nacional de Saúde n ${ }^{\circ} 466$, de dezembro de 2012, tendo o projeto sido aprovado pela Comissão Científica e pelo Comitê de Ética em Pesquisa (CEP), sob parecer 3.554.625.

O campo de estudo foi o hospital de ensino Santa Casa de Misericórdia, situado na região norte do Ceará e considerado um hospital de referência em urgência e emergência no município de Sobral. A instituição não trabalha com a ELPO na SAEP, sendo caracterizada como de nível terciário e alta complexidade e atuando como hospital-escola, com atenção voltada para o desenvolvimento de ensino, pesquisa, extensão e assistência à saúde. O CC dispõe de 10 salas operatórias (SO) que funcionam 24 horas por dia, com cirurgias de emergências, urgências e eletivas, feitas por convênios ou pelo Sistema Único de Saúde (SUS).

A pesquisa foi realizada com pacientes atendidos na Unidade de Centro Cirúrgico do Hospital Santa Casa de Misericórdia de Sobral. A população foi composta de pessoas de ambos os sexos admitidas no CC com necessidades de abordagem cirúrgica, fosse do tipo de emergência, fosse de urgência ou eletiva. Os critérios de inclusão considerados na pesquisa foram:

- paciente submetido a cirurgia de trauma músculo-esquelético;

- pacientes com necessidade de reabordagem cirúrgica decorrente do trauma;

- idade igual ou superior a 18 anos;

- pacientes conscientes;

- pacientes inconscientes com familiar acompanhante que autorizasse a realização da pesquisa.

A coleta de dados foi realizada no período de outubro e novembro de 2019, sendo a amostra constituída de 31 pacientes. O trabalho considerou dois instrumentos: perfil clínico-epidemiológico e ELPO.

O instrumento do perfil clinico-epidemiológico foi utilizado para a realização de uma entrevista semiestruturada com cada paciente no intuito de identificar características sociais e epidemiológicas, o que auxilia na identificação dos fatores de risco presentes e das possibilidades de orientações para intervenções. Já a ELPO foi aplicada como instrumento de investigação da situação clínica referente ao posicionamento cirúrgico na SO. As aplicações desses dois instrumentos foram necessárias para o melhor delineamento deste trabalho. 
No processo de coleta de dados, houve o primeiro contato com o paciente ou familiar para fornecer explicações sobre o estudo. Após a assinatura do termo de consentimento, empregou-se a entrevista estruturada, com o preenchimento do instrumento do perfil clínico-epidemiológico e, em seguida, acompanhamento do paciente na SO, onde foi aplicada a ELPO no período transoperatório. A escala foi aplicada após o paciente ser posicionado na mesa operatória para a cirurgia.

A análise foi realizada com os resultados obtidos pelos instrumentos estatísticos descritivos. O perfil clínico-epidemiológico e os dados da ELPO foram analisados no software Statistical Package for the Social Sciences (SPSS). Os resultados estão exibidos em formato de gráficos e tabelas, que estabelecem visualização e compreensão rápidas.

\section{RESULTADOS}

As análises e discussões referentes à aplicação da ELPO em 31 pacientes cirúrgicos, nos períodos pré-operatório e transoperatório, estão descritas em tópicos e organizadas em perfil clínico-epidemiológico (sexo, raça, faixa etária, causas de internação) e avaliação do escore de risco, segundo a ELPO.

A maioria dos pacientes $(27 / 87,1 \%)$ era do sexo masculino e autointitulada de cor parda (28/90,3\%); $17(54,8 \%)$ estavam na faixa etária entre 18 e 39 anos e 11 (35,4\%) entre 40 e 59 anos (Gráfico 1).

No Gráfico 2, verifica-se que, entre as causas de internação dos pacientes do estudo, houve predomínio de acidentes motociclísticos (16/51,6\%), seguidos de acidentes de trabalho e queda da própria altura (4/12,9\% para cada ocorrência).

Em relação ao tipo de especialidade cirúrgica, houve maior frequência de procedimentos de cirurgia plástica $(14 / 45,2 \%)$, seguida pela ortopedia/traumatologia (9/29\%) e neurologia $(3 / 9,6 \%)$.

Na Tabela 1, destacam-se os procedimentos cirúrgicos aos quais os 31 pacientes foram submetidos, com destaque

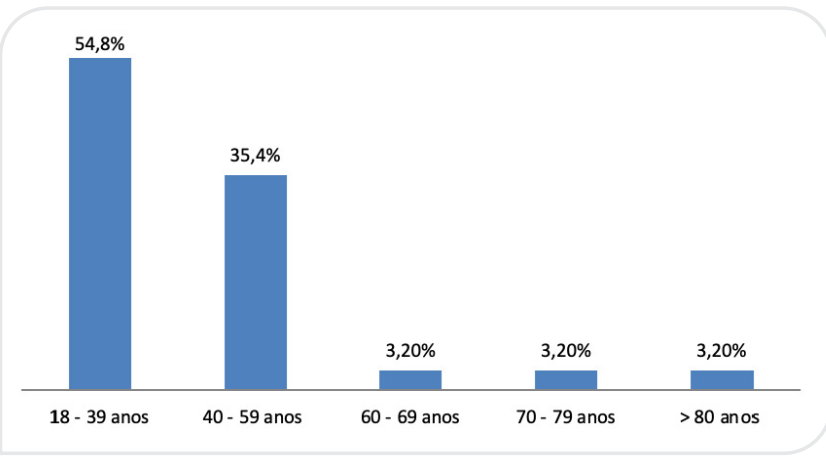

Gráfico 1. Faixa etária dos pacientes do estudo. para os debridamentos (9/29\%), seguidos de redução cirúrgica de fratura $(4 / 12,9 \%)$.

A Tabela 2 traz a análise das sete variáveis da ELPO aplicadas aos pacientes da amostra do estudo. Houve prevalência da posição supina $(26 / 83,8 \%)$, tempo de duração da cirugia de até uma hora $(21 / 67,7 \%)$, anestesia local $(18 / 58 \%)$. Todos os pacientes (31/100\%) utilizaram, como superfície de suporte, o colchão da mesa cirúrgica e coxins de espuma. Quanto à posição dos membros, a maioria $(21 / 67,7 \%)$ teve abertura dos membros superiores em ângulo menor do que 90 graus. A grande maioria dos pacientes não tinha comorbidades $(30 / 96,7 \%)$ e a faixa etária predominante foi de adultos jovens, dos 18 aos 39 anos (17/54,8\%), seguidos da faixa etária entre 40 e 59 anos (11/35,4\%).

Diante da distribuição dos escores de cada item da ELPO (Tabela 2), observou-se que todos os 31 (100\%) pacientes participantes do estudo tiveram escores menores ou iguais a 19 , classificados, portanto, como baixo risco de desenvolver lesão por conta do posicionamento cirúrgico no perioperatório (Tabela 3).

\section{DISCUSSÃO}

A prevalência das ocorrências na população masculina ocorre pela sua maior exposição às atividades econômicas e recreativas de risco, como também à violência e às drogas, comportamento específico do sexo, além da influência de fatores culturais e sociais ${ }^{11,12}$.

Quanto à raça, verificou-se um predomínio de pessoas pardas $(90,3 \%)$. Quando lhes foi solicitado identificarem sua raça, alguns pacientes tiveram dificuldade em responder. A raça é representada pela cor de pele, autodeclarada nos principais censos brasileiros realizados pelo Instituto Brasileiro de Geografia e Estatística (IBGE), e é caracterizada como um grupo de indivíduos que compartilham certas características ${ }^{13-15}$.

No que se refere à faixa etária, neste estudo houve predomínio de adultos jovens ( $54,8 \%$ entre 18 e 39 anos). O paciente mais jovem tinha 18 anos e o mais velho, 83. Corroborando o estudo ${ }^{11}$, quando analisada a faixa etária, a população de adultos jovens é a mais acometida por casos de violência, havendo diversas teorias sobre os motivos para isso, como inexperiência, impulsividade, irresponsabilidade, estilo de vida, busca de situações de risco para satisfação pessoal e abuso de substâncias psicoativas ${ }^{11}$. No Brasil, existem sérios problemas de exclusão juvenil e desigualdade social que contribuem para este número expressivo de vítimas jovens ${ }^{12}$.

Quando avaliadas as causas de internação, verifica-se a predominância de acidentes com motocicletas $(51,60 \%)$, seguidos de acidentes de trabalho e queda da própria altura $(12,9 \%$ cada 
um). As causas de internação no estudo são condizentes com as características do local de pesquisa, um hospital público, de grande porte, especializado em trauma, que atende pessoas, em sua maioria, vítimas de acidentes de trânsito.

Em relação ao tipo de especialidade cirúrgica, as mais frequentes foram cirurgias plásticas $(45,16 \%)$ e ortopédicas por traumas (29,0\%). Há divergência com outros estudos ${ }^{15,16}$ que tiveram como população alvo pacientes submetidos a procedimentos eletivos de qualquer especialidade cirúrgica.

Estudo realizado em um hospital de reabilitação em Brasília $(\mathrm{DF})^{16}$ encontrou prevalência da especialidade cirúrgica de ortopedia $(48,1 \%)$, seguida de neurocirurgia $(36,8 \%)$ e cirurgia plástica $(11,3 \%)$. Em outra pesquisa, realizada em Minas Gerais ${ }^{15}$, houve predomínio de cirurgias do aparelho digestório $(33,7 \%)$, cabeça/ pescoço e ortopedia (13,5\% cada) e otorrinolaringologia $(11,2 \%)$.

Em relação ao tipo de procedimento cirúrgico, pode-se constatar que, nos casos traumáticos, a maior incidência de cirurgias foi debridamento (29\%), seguido de redução de fratura $(12,9 \%)$. Outro estudo ${ }^{15}$ mostrou que as maiores prevalências de cirurgias foram mamárias $(33,7 \%)$, buco-maxilares $(13,5 \%)$ e otorrinolaringológicas $(11,2 \%)$. Porém, há que se considerar as diferenças entre ambas as instituições.

$\mathrm{Na}$ análise das variáveis da ELPO, quanto à posição em que os pacientes permaneceram, a posição supina teve maior incidência $(83,8 \%)$. Nessa posição, deve-se ter o cuidado de proteger as proeminências do pacientes em contato com a mesa cirúrgica, pois, com o tempo prolongado e a imobilização, eles podem desenvolver lesão por pressão nas regiões occipital, ombros, escápulas, cotovelos, lombar e sacral, glútea e calcanhar. Além disso, a frequência cardíaca, a resistência vascular e a capacidade pulmonar podem ser diminuídas na posição supina ${ }^{4}$.

Com relação ao tempo cirúrgico, houve prevalência de procedimentos com pequena duração, de até uma hora (67,7\%). O tempo de cirurgia de até uma hora resulta no escore 1 da ELPO.

Quanto ao tipo de anestesia, dos 31 pacientes estudados na amostra investigada, $58 \%$ foram submetidos a anestesia local,

Tabela 1. Procedimentos cirúrgicos aos quais os pacientes do estudo foram submetidos.

\begin{tabular}{|l|c|c|}
\hline Procedimento cirúrgico & Número & $\begin{array}{c}\text { Percentagem } \\
(\%)\end{array}$ \\
\hline Debridamento & 9 & 29 \\
\hline Redução cirúrgica de fratura & 4 & 12,9 \\
\hline Amputação & 2 & 6,5 \\
\hline Drenagem liquórica lombar & 2 & 6,5 \\
\hline Osteotomia & 2 & 6,5 \\
\hline Reparação de perda de substância & 2 & 6,5 \\
\hline Toracostomia com drenagem & 2 & 6,5 \\
\hline Artroplastia & 1 & 3,2 \\
\hline Cervicotomia & 1 & 3,2 \\
\hline Cranioplastia & 1 & 3,2 \\
\hline Enxerto & 1 & 3,2 \\
\hline Gastrostomia & 1 & 3,2 \\
\hline Impantação de cateter & 1 & 3,2 \\
\hline Reconstrução parcial & 1 & 3,2 \\
\hline Tratamento cirúrgico de fratura & 1 & 3,2 \\
\hline Total & 31 & 100 \\
\hline
\end{tabular}

\section{$51,60 \%$}

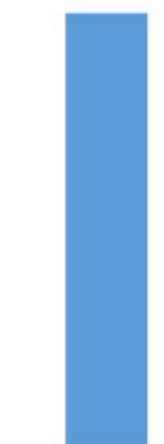

$12,9 \%$ $12,9 \%$
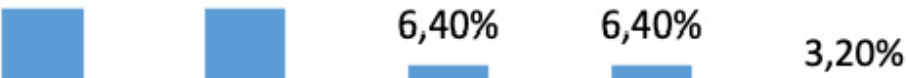

$3,20 \%$

$3,20 \%$

\section{ACIDENTE ACIDENTE QUEDA DA AGRESSÃO FERIMENTO QUEDA DE QUEDA DA TENTATIVA DE MOTO DE PRÓPRIA FÍSICA PORARMA BICICLETA BRANCA \\ DE SUICÍDIO}

Gráfico 2. Causas de internação dos pacientes do estudo. 
sendo essa a maior frequência de tipo de anestesia utilizado nos procedimentos cirúrgicos. A anestesia compreende uma condição temporária de perda de sensibilidade e, dependendo do seu tipo, podem ocorrer complicações, perante as quais são necessárias ações que envolvem grande responsabilidade dos profissionais na prestação do cuidado seguro. O tempo prolongado do processo anestésico-cirúrgico altera a conformação da pele e pode acarretar ao paciente alguns prejuízos e aumento do risco de lesões em decorrência do posicionamento ${ }^{17}$.

Em relação à distribuição das superfícies de suporte utilizadas para o posicionamento dos pacientes cirúrgicos no hospital, para todos eles (100\%) se utilizaram o colchão da mesa cirúrgica de espuma convencional e coxins de espuma. O CC do estudo é composto de $10 \mathrm{SO}$, nas quais se utiliza essa superfície de suporte em todas as mesas cirúrgicas, oferecendo maior proteção ao paciente e menor risco de desenvolver lesão. Esse achado corrobora um estudo ${ }^{16}$ no qual houve prevalência do uso de superfície de suporte do colchão de mesa cirúrgica de espuma convencional e coxins de espuma com a maioria dos pacientes $(59,4 \%)$.
Quanto à posição dos pacientes na mesa cirúrgica no período intraoperatório, a mais prevalente $(21 / 67,7 \%)$ foi a abertura dos membros superiores $<90^{\circ}$. O posicionamento cirúrgico é um procedimento de responsabilidade da equipe

Tabela 3. Pacientes do estudo, segundo o escore da Escala de Avaliação de Risco para o Desenvolvimento de Lesões Decorrentes do Posicionamento Cirúrgico.

\begin{tabular}{|l|c|c|}
\hline ELPO & $\mathbf{n}$ & $\%$ \\
\hline Baixo risco $(\leq 19)$ & 31 & 100 \\
\hline 9 & 4 & 12,9 \\
\hline 10 & 4 & 12,9 \\
\hline 11 & 6 & 19,3 \\
\hline 12 & 5 & 16,1 \\
\hline 13 & 4 & 12,9 \\
\hline 14 & 6 & 19,3 \\
\hline 16 & 1 & 3,20 \\
\hline 19 & 1 & 3,20 \\
\hline Alto risco $(>19)$ & 0 & 0 \\
\hline
\end{tabular}

Tabela 2. Análise descritiva das variáveis da Escala de Avaliação de Risco para o Desenvolvimento de Lesões Decorrentes do Posicionamento Cirúrgico, aplicada aos pacientes do estudo.

\begin{tabular}{|c|c|c|c|}
\hline & Variáveis & $\mathbf{N}$ & $\%$ \\
\hline \multirow{2}{*}{ Posição cirúrgica } & Supina & 26 & 83,8 \\
\hline & Lateral & 5 & 16,1 \\
\hline \multirow{3}{*}{ Tempo de cirurgia } & Até $1 \mathrm{~h}$ & 21 & 67,7 \\
\hline & Acima de 1 e até $2 \mathrm{~h}$ & 9 & 29 \\
\hline & Acima de 2 e até $4 \mathrm{~h}$ & 1 & 3,2 \\
\hline \multirow{4}{*}{ Tipo de anestesia } & Local & 18 & 58 \\
\hline & Sedação & 3 & 9,6 \\
\hline & Regional & 8 & 25,8 \\
\hline & Geral & 2 & 6,5 \\
\hline Superfície de suporte & Colchão de mesa cirúrgica de espuma + coxins de espuma & 31 & 100 \\
\hline \multirow{4}{*}{ Posição dos membros } & Alinhamento corporal & 6 & 19,3 \\
\hline & Abertura dos membros superiores $<90^{\circ}$ & 21 & 67,7 \\
\hline & Elevação dos joelhos $<90^{\circ}$ e abertura dos membros inferiores $<90^{\circ}$ & 1 & 3,2 \\
\hline & Elevação dos joelhos $>90^{\circ}$ ou abertura dos membros inferiores $>90^{\circ}$ & 3 & 9,6 \\
\hline \multirow{2}{*}{ Comorbidades } & Sem comorbidades & 30 & 96,7 \\
\hline & Diabetes Mellitus & 1 & 3,2 \\
\hline \multirow{5}{*}{ Idade } & $18-39$ anos & 17 & 54,8 \\
\hline & $40-59$ anos & 11 & 35,4 \\
\hline & $60-69$ anos & 1 & 3,2 \\
\hline & $70-79$ anos & 1 & 3,2 \\
\hline & $>80$ anos & 1 & 3,2 \\
\hline
\end{tabular}


cirúrgica que deve ser realizado com cuidado, de forma segura, e destinado especificamente para cada tipo de paciente, pois implica risco durante a assistência ${ }^{16}$.

No que diz respeito às comorbidades específicas elencadas na ELPO, apenas um paciente tinha diabetes mellitus e teve maior escore nesse quesito da Escala. A grande maioria dos pacientes do estudo $(96,7 \%)$ não tinha nenhuma patologia preexistente/comorbidade. Na pesquisa mineira ${ }^{15}$, em relação às doença preexistentes, a maior parte dos pacientes $(48,3 \%)$ também não apresentou comorbidades, corroborando os achados deste trabalho.

Na variável faixa etária da ELPO, a maioria dos pacientes do presente estudo $(54,8 \%)$ tinha entre 18 e 39 anos, o que favoreceu o menor risco de desenvolvimento de lesões. Esse achado está em consonância com outra pesquisa ${ }^{15}$, que obteve a mesma faixa etária como mais prevalente (34,8\%). Estudos demonstram que a incidência de complicações aumenta proporcionalmente à idade, por conta da diminuição da tolerância ao posicionamento prolongado, assim como se encontra na população obesa, pois, dependendo do tipo de posição, ela favorece a compressão do diafragma e dificulta a expansibilidade torácica ${ }^{18,19}$.

Na mensuração do risco de ocorrência de lesões decorrentes do posicionamento cirúrgico, a pontuação da ELPO demonstrou que todos os pacientes da amostra (31/100\%) foram classificados como baixo risco (pontuações menores ou iguais a 19), visto que foram posicionados em supina $(83,80 \%)$, tiveram suas cirurgias realizadas num tempo de até uma hora $(67,7 \%)$, foram submetidos a anestesia local $(58 \%)$, não tinham comorbidades $(96,7 \%)$ e pertenciam à faixa etária dos 18 aos 39 anos (54,8\%). Para cada um desses itens das variáveis mensuradas na ELPO, a pontuação equivale a um ponto (escore $=1$ ), o que resultou em escores totais baixos (escore total $\leq 19$ ), traduzidos por menor risco de desenvolver lesão resultante do posicionamento perioperatório.

Portanto, a incidência de lesões decorrentes do posicionamento cirúrgico do presente estudo foi considerada baixa quando comparada às de outros estudos ${ }^{15,16}$. Em outra pesquisa ${ }^{15}$, também houve maior prevalência de pacientes com baixo escore de risco $(53,9 \%)$, porém quase metade deles $(46,1 \%)$ apresentou escore de risco ELPO $>19$, que corresponde ao maior risco de desenvolvimento de lesões relacionadas ao posicionamento cirúrgico. Em contrapartida, os autores de uma pesquisa ${ }^{16}$ encontraram predominantemente pacientes com maior risco de desenvolver lesões por posicionamento perioperatório $(54,7 \%)$ em comparação àqueles com menor risco $(45,3 \%)$. Mais uma vez, há que se considerar as características da instituição, assim como o tipo de clientela que ela atende.
Diante desses resultados, ressalta-se a importância da assistência prestada no $\mathrm{CC}$, mais especificamente na $\mathrm{SO}$, com a disponibilização de diferentes recursos para o posicionamento adequado, enfermeiros acompanhando o paciente e o procedimento, realizando a SAEP e sendo responsáveis diretos, com os demais membros da equipe, pelo posicionamento do paciente para a cirurgia.

De todas as formas, é possível verificar a eficácia da utilização da ELPO ao se propor a avaliação de sete itens específicos, porém intimamente interligados entre si, cuja somatória total/ escore final significa a propensão que cada paciente avaliado tem de apresentar ou não lesão decorrente da posição cirúrgica.

\section{CONCLUSÃO E CONSIDERAÇÕES FINAIS}

A proposta do estudo possibilitou a aplicação da ELPO em um CC hospitalar do Nordeste do Brasil, onde não se utilizava o instrumento da ELPO na SAEP.

O estudo proporcionou a avaliação dos seus itens com consequente revisão de evidências científicas e oportunizou conhecer o perfil dos pacientes submetidos a procedimentos cirúrgicos na instituição, caracterizando-os quanto ao risco de desenvolver lesões resultantes da posição cirúrgica. Os 31 pacientes acompanhados nos períodos pré e transoperatório foram classificados como baixo risco de desenvolver tais lesões, uma vez que os escores da ELPO foram menores ou iguais a 19.

Tais resultados tornaram inviável a implementação dos cuidados modificáveis na prevenção de lesões no ato do posicionamento cirúrgico, pois não houve pacientes com alto risco de desenvolver lesões. Os cuidados modificáveis seriam aplicados no paciente que apresentasse escore de alto risco. À equipe cirúrgica, durante o período intraoperatório, caberia efetuar cuidados relacionados: transições no tipo da posição cirúrgica, na superfície de suporte, acrescentando os coxins (algodão, espuma ou viscoelástico) ou suporte com acolchoamento, ou, ainda, alterar a posição dos membros do paciente no intuito de promover conforto na mesa cirúrgica e evitar possíveis lesões.

Ressalta-se que, no referido hospital, os cuidados da equipe durante o posicionamento favoreceram o baixo risco de lesões, demonstrando a qualidade da assistência prestada aos pacientes cirúrgicos.

Como relevantes contribuições deste estudo para a área da enfermagem cirúrgica, no campo científico e na prática clínica, foram obtidos bons resultados na assistência ao paciente. Os autores sugerem que outras pesquisas sejam realizadas, com a aplicação da ELPO em pacientes de outras especialidades cirúrgicas, variações de idade e avaliação dos dispositivos de proteção. 


\section{REFERÊNCIAS}

1. Andrade LEL, Lopes JM, Souza Filho MCM, Vieira Júnior RF, Farias LPC, Santos CCM, et al. Cultura de segurança do paciente em três hospitais brasileiros com diferentes tipos de gestão. Ciênc Saúde Coletiva. 2018;23(1):161-72. http://dx.doi. org/10.1590/1413-81232018231.24392015

2. Carlesi KC, Padilha KG, Toffoletto MC, Henriquez-Roldán C, Juan MAC. Ocorrência de incidentes de segurança do paciente e carga de trabalho de enfermagem. Rev Latino-Am Enferm. 2017;25:e2841. http://dx.doi.org/10.1590/1518-8345.1280.2841

3. Oliveira MCB, Korb A, Zocche DAA, Cabral DB, Pertille F, Frigo J. Adesão do checklist cirúrgico à luz da cultura de segurança do paciente. Rev SOBECC. 2018:23(1):36-42. https://doi.org/10.5327/Z1414-4425201800010007

4. Carvalho R, Moraes MW. A inserção do centro cirúrgico no contexto hospitalar. In: Carvalho R, Bianchi ERF, editores. Enfermagem em centro cirúrgico e recuperação. $2^{\mathrm{a}}$ ed. Barueri: Manole; 2016.

5. Miranda AB, Fogaça AR, Rizzetto M, Lopes LCC. Posicionamento cirúrgico: cuidados de enfermagem no transoperatório. Rev SOBECC. 2016;21(1):52-8. https://doi.org/10.5327/Z1414-4425201600010008

6. North American Nursing Diagnosis Association (NANDA I). Diagnósticos de Enfermagem da NANDA: definições e classificação 2018-2020. Porto Alegre: Artmed; 2018.

7. Lopes CMM. Escala de avaliação de risco para o desenvolvimento de lesões decorrentes do posicionamento cirúrgico: construção e validação [tese online]. Ribeirão Preto: Escola de Enfermagem, Universidade de São Paulo; 2013 [acessado em 13 nov. 2018]. Disponível em: https:// www.teses.usp.br/teses/disponiveis/22/22132/tde21052014184456/ publico/CamilaMendoncadeMoraesLopes.pdf

8. Associação Brasileira de Enfermeiros de Centro Cirúrgico, Recuperação Anestésica e Centro de Material de Esterilização (SOBECC). Diretrizes de práticas em enfermagem cirúrgica e processamento de produtos para a saúde. 7ª ed. São Paulo: SOBECC; 2017.

9. Heizenroth PA. Posicionamento do paciente para cirurgia. In: Rothrock JCA, editor. Cuidados de enfermagem ao paciente cirúrgico. 13a ed. Rio de Janeiro: Mosby Elsevier; 2007. p. 130-57.

10. Lopes CMM. Posicionamento cirúrgico: evidências para o cuidado de enfermagem [dissertação online]. Ribeirão Preto: Escola de Enfermagem, Universidade de São Paulo; 2009 [acessado em 13 nov. 2018]. Disponível em: http://biblioteca.posgraduacaoredentor.com.br/link/?id=2359917
11. Carvalho IC, Saraiva IS. Perfil das vítimas de trauma atendido pelo serviço de atendimento móvel de urgência. Rev Interdisciplinar [Internet]. 2015 [acessado em 13 nov. 2018]:8(1):137-48. Disponível em: https://revistainterdisciplinar.uninovafapi.edu.br/index.php/ revinter/article/view/392/pdf_192

12. Alves WA. Análise da ocorrência dos óbitos por agressão a partir dorelacionamento das bases de dados do Ministério da Saúde e da Defesa Social em Maceió, Alagoas, no início do século XXI [tese]. Maceió: Fundação Oswaldo Cruz; 2014.

13. Moretto MC, Fontaine AM, Garcia CAMS, Neri AL, Guariento ME. Associação entre cor/raça, obesidade e diabetes em idosos da comunidade: dados do Estudo FIBRA. Cad Saúde Pública. 2016;32(10):e00081315. https://doi.org/10.1590/0102-311X00081315

14. Instituto Brasileiro de Geografia e Estatística (IBGE). Características étnico-raciais da população. Um estudo das categorias de classificação de cor ou raça [Internet]. Instituto Brasileiro de Geografia e Estatística 2008 [acessado em 12 set. 2018]. Disponível em: http://www. ibge.gov.br/home/estatistica/populacao/caracteristicas_raciais/ PCERP2008.pdf

15. Caetano EPS. Risco para lesões do posicionamento cirúrgico decorrentes da posição supina [dissertação online]. Minas Gerais: Escola de Enfermagem, Universidade Federal de Minas Gerais; 2018 [acessado em 15 fev. 2020]. Disponível em: https://repositorio.ufmg. $\mathrm{br} /$ bitstream/1843/ENFC-B9AHDX/1/ricapatriciasouzacaetano.pdf

16. Nascimento FCL. Aplicação da escala de risco para lesão no posicionamento cirúrgico em hospital de reabilitação [dissertação online]. Brasília: Faculdade de Ciências da Saúde, Universidade de Brasilia; 2018 [acessado em 17 fev. 2020]. Disponível em: https:// repositorio.unb.br/handle/10482/33851

17. Lima PR, Damacena DEL, Neves VLS, Campos RBN, Silva FAA, Bezerra SMG. Ocorrência de lesão por pressão em pacientes hospitalizados: uma revisão integrativa. Uningá Rev J. 2017;32(1):53-67.

18. Lopes $C M$, Galvão $C M$. Posicionamento cirúrgico: evidências para o cuidado de enfermagem. Rev Latino-Am Enferm. 2010;18(2):28794. https://doi.org/10.1590/S0104-11692010000200021

19. Grigoleto ARL, Avelar MCQ, Lacerda RA, Mendonça SHF. Complicações decorrentes do posicionamento cirúrgico de clientes idosos submetidos à cirurgia de quadril. Esc Anna Nery. 2011;15(3):531-5. https://doi. org/10.1590/S1414-81452011000300013 